\title{
ASSOCIATIVE AND JORDAN SHIFT ALGEBRAS
}

\author{
OTTMAR LOOS AND ERHARD NEHER
}

(Communicated by Maurice Auslander)

\begin{abstract}
Let $R$ be the shift algebra, i.e., the associative algebra presented by generators $u, v$ and the relation $u v=1$. As $\mathrm{N}$. Jacobson showed, $R$ contains an infinite family of matrix units. In this paper, we describe the Jordan algebra $R^{+}$and its unital special universal envelope by generators and relations. Moreover, we give a presentation for the Jordan triple system defined on $R$ by $P_{x} y=x y^{*} x$ where ${ }^{*}$ is the involution on $R$ with $u^{*}=v$. As a consequence, we prove the existence of an infinite rectangular grid in a Jordan triple system $V$ containing tripotents $c$ and $d$ with $V_{2}(c)=V_{2}(d) \oplus\left(V_{2}(c) \cap V_{1}(d)\right)$ and $V_{2}(c) \cap V_{1}(d) \neq 0$.
\end{abstract}

\section{THE SHIFT ALGEBRA}

Let $k$ be a commutative ring and $R$ the associative unital $k$-algebra presented by generators $u, v$ and the relation $u v=1$. We call $R$ the shift algebra since it has a faithful representation on $k^{(\infty)}=\sum_{i=0}^{\infty} k \cdot \varepsilon_{i}$ by the shift operators

$$
\begin{aligned}
& u: \varepsilon_{i} \mapsto \varepsilon_{i-1}, \varepsilon_{0} \mapsto 0, \\
& v: \varepsilon_{i} \mapsto \varepsilon_{i+1} .
\end{aligned}
$$

In [1] Jacobson essentially proved the following theorem on the structure of $R$.

Theorem 1. (a) $R$ is a free $k$-module with basis $1, u^{n}, v^{n} \quad(n \geq 1)$, and $e_{i j}=$ $v^{i}(1-v u) u^{j} \quad(i, j \geq 0)$.

(b) The $e_{i j}$ satisfy the multiplication table for matrix units.

(c) The subspace $E$ spanned by the $e_{i j}$ is an ideal, namely, the ideal generated by $e_{00}=1-v u$, and $R / E \cong k\left[X, X^{-1}\right]$ (Laurent polynomials).

We remark that $R$ has an involution ${ }^{*}$ with $u^{*}=v$, but the elements $u$ and $v$ of $R$ obviously do not play symmetrical roles; in particular, there is no automorphism of $R$ interchanging $u$ and $v$ and no involution fixing the generators. In this note we introduce a symmetric shift algebra $S$ on two generators defined by left-right symmetric relations and, hence, carrying an involution $\pi$ fixing the generators. The defining relations of $S$ are expressible by Jordan products. This leads us to define a Jordan shift algebra $J$ and a Jordan shift triple system $T$. We show that $J \cong R^{+}$is special with unital special universal

Received by the editors August 26, 1991 and, in revised form, April 14, 1992.

1991 Mathematics Subject Classification. Primary 17C10; Secondary 16A10, 16A42, 17 C05.

The second author's research was partially supported by an NSERC operating grant. 
envelope $(S, \pi)$ and that $T$ is isomorphic to $R$ with the Jordan triple product $P_{x} y=x y^{*} x$. As a consequence we obtain the existence of an infinite set of orthogonal idempotents in a unital Jordan algebra containing a noninvertible regular pair $x, y$ (i.e., $U_{x} y=x, U_{y} x=y$ ) with vanishing Bergmann operators $W(x, y)\left(=\mathrm{Id}-V_{x, y}+U_{x} U_{y}\right)$ and $W(y, x)$, and the existence of an infinite rectangular grid in a Jordan triple system $V$ containing tripotents $c$ and $d$ with the property that $V_{2}(c)=V_{2}(d) \oplus\left(V_{2}(c) \cap V_{1}(d)\right)$ and $V_{2}(c) \cap V_{1}(d) \neq 0$.

Standard references for notation and terminology are $[2,5]$.

\section{THE SYMMETRIC SHIFT ALGEBRA}

The symmetric shift algebra is the associative unital $k$-algebra $S$ presented by generators $s, t$ and relations

$$
\begin{gathered}
s=s t s, \quad t=t s t, \\
(1-s t) t^{n}(1-t s)=(1-t s) s^{n}(1-s t)=0 \quad \text { for all } n \geq 0 .
\end{gathered}
$$

From the definition it is clear that $S$ has an involution $\pi$ and an automorphism $\rho$ of period 2, commuting with each other, and acting on the generators by

$$
s^{\pi}=s, \quad t^{\pi}=t, \quad s^{\rho}=t, \quad t^{\rho}=s .
$$

Hence ${ }^{*}=\pi \circ \rho=\rho \circ \pi$ is an involution with $s^{*}=t$. In the sequel, the locution "by symmetry" will mean "by an application of $\pi, \rho$, or *".

Theorem 2. (a) $S$ is a free $k$-module with basis $1, s^{n}, t^{n} \quad(n \geq 1)$, and $g_{i j}=$ $s^{i}(1-s t) t^{j}, h_{i j}=t^{i}(1-t s) s^{j} \quad(i, j \geq 0)$.

(b) The $g_{i j}$ and $h_{i j}$ satisfy the multiplication table for matrix units and annihilate each other.

(c) The subspaces $G, H$ spanned by the $g_{i j}, h_{i j}$, respectively, are ideals, namely, the ideals generated by $g_{00}=1-s t, h_{00}=1-t s$, respectively. They are interchanged by $\pi$ and $\rho$, with $S / G \cong R \cong S / H$ and $S /(G \oplus H) \cong k\left[X, X^{-1}\right]$.

(d) $S$ has a faithful representation on $k^{(\infty)} \oplus k^{(\infty)}=\sum_{i=0}^{\infty} k \varepsilon_{i} \oplus k \eta_{i}$ via $s\left(\varepsilon_{i}\right)=\left(1-\delta_{i o}\right) \varepsilon_{i-1}, s\left(\eta_{i}\right)=\eta_{i+1}, t\left(\varepsilon_{i}\right)=\varepsilon_{i+1}, t\left(\eta_{i}\right)=\left(1-\delta_{i o}\right) \eta_{i-1}$.

Proof. We have $g_{i j}^{\pi}=h_{j i}=g_{j i}^{\rho}$; hence, $G^{\pi}=G^{\rho}=H$. Let us set $g_{p q}=0$ and $h_{p q}=0$ if $p$ or $q$ is negative.

First we show

$$
\begin{gathered}
g_{i j} h_{k l}=0, \\
t^{i} s^{j}=\left\{\begin{array}{cc}
t^{i-j}-\sum_{k=1}^{j} h_{i-k, j-k} & \text { if } i>j, \\
s^{j-i}-\sum_{k=1}^{j} h_{i-k, j-k} & \text { if } i \leq j,
\end{array}\right. \\
s^{n} g_{i j}=g_{i+n, j}, \quad t^{n} g_{i j}=g_{i-n, j}, \\
g_{i j} s^{n}=g_{i, j-n}, \quad g_{i j} t^{n}=g_{i, j+n}, \\
g_{i j} g_{k l}=\delta_{j k} g_{i l} .
\end{gathered}
$$

Indeed, (3) follows from (2), while (4) and the first formula in (5) are clear from the definitions. For the second one, note in case $n>i$ that $t^{n} g_{i j}=t^{n} s^{i} g_{0 j}=$ $t^{n-i} g_{0 j}$ (by (3) and (4)) $=0$ by (1) and in case $n \leq i$ that $t^{n} g_{i j}=t^{n} s^{i} g_{0 j}=$ $s^{i-n} g_{0 j}=g_{i-n, j}$ by (3) and (4). 
By symmetry we have (6). The multiplication table (7) for the $g_{i j}$ is now an easy consequence of the shift formulas (5) and (6):

$$
\begin{aligned}
g_{i j} g_{k l} & =g_{i j} s^{k}(1-s t) t^{l}-g_{i, j-k} g_{0 l} \\
& =s^{i}(1-s t) t^{j} g_{k l}=g_{i 0} g_{k-j, l} .
\end{aligned}
$$

Thus if $j \neq k$ then either $g_{i, j-k}=0$ or $g_{k-j, l}=0$, whereas for $j=k$ we get $g_{i j} g_{j l}=g_{i 0} g_{0 l}=g_{i l}$ by $(1-s t)^{2}=1-s t$. The multiplication table for the $h_{i j}$ is obtained by symmetry.

From (3)-(7) and the symmetrical formulas it follows that the linear span of the $g_{i j}, h_{i j}$ and the powers of $s$ and $t$ is a subalgebra of $S$ and thus is all of $S$ and that $G$ and $H$ are ideals of $S$ annihilating each other. By the universal property of $S$ we have surjective homomorphisms $\varphi_{1}, \varphi_{2}: S \rightarrow R$ given by $\varphi_{1}(s)=u, \varphi_{1}(t)=v$ and $\varphi_{2}(s)=v, \varphi_{2}(t)=u$ respectively. The homomorphism $\varphi=\varphi_{1} \oplus \varphi_{2}: S \rightarrow R \oplus R$ then maps $s^{n} \mapsto u^{n} \oplus v^{n}, t^{n} \mapsto$ $v^{n} \oplus u^{n}, \quad g_{i j} \mapsto 0 \oplus e_{i j}, h_{i j} \mapsto e_{i j} \oplus 0$. Hence (a) follows from Theorem $1(\mathrm{a})$. Since $\operatorname{ker}\left(\varphi_{1}\right)=G$ and $\operatorname{ker}\left(\varphi_{2}\right)=H$, we have $S / G \cong R \cong S / H$. Also $(G+H) / G \cong E \cong(G+H) / H$, whence $S /(G \oplus H) \cong R / E \cong k\left[X, X^{-1}\right]$. If $\psi$ denotes the representation of $R$ on $k^{(\infty)}$ mentioned in $\S 1$ then $(\psi \oplus \psi) \circ \varphi$ is a representation of $S$ as claimed in (d). This completes the proof.

\section{THE JORDAN SHIFT ALGEBRA}

Observe that (1) and (2) make sense in a special Jordan algebra since $s t s=$ $U_{s} t$ and $(1-s t) z(1-t s)=z-V_{s, t} z+U_{s} U_{t} z=W(s, t) z$. We therefore define the Jordan shift algebra as the unital quadratic Jordan algebra $J$ over $k$ presented by generators $a, b$ and relations

$$
\begin{gathered}
a=U_{a} b, \quad b=U_{b} a, \\
W(a, b) b^{n}=W(b, a) a^{n}=0 \text { for all } n \geq 0 .
\end{gathered}
$$

These relations are symmetric in $a$ and $b$. Hence there is an automorphism $\tau$ of period two of $J$ interchanging $a$ and $b$.

Theorem 3. The assignment $a \mapsto u, b \mapsto v$ induces an isomorphism $\varphi: J \rightarrow R^{+}$ (the Jordan algebra associated to $R$ ), which is compatible with $\tau$ and ${ }^{*}$.

Proof. Clearly, $\varphi$ is compatible with $\tau$ and ${ }^{*}$ and maps $a^{n}$ and $b^{n}$ respectively to $u^{n}$ and $v^{n}$. Define $c_{i j} \in J$ by

$$
c_{i j}= \begin{cases}a^{j} \circ b^{i}-a^{j+1} \circ b^{i+1} & \text { if } i, j \geq 0, \\ 0 & \text { if } i<0 \text { or } j<0 .\end{cases}
$$

Then

$$
\begin{aligned}
\varphi\left(c_{i j}\right) & =u^{j} v^{i}+v^{i} u^{j}-u^{j+1} v^{i+1}-v^{i+1} u^{j+1} \\
& =u^{j}(1-u v) v^{i}+v^{i}(1-v u) u^{j}=0+e_{i j} .
\end{aligned}
$$

Therefore the theorem will follow from Theorem 1 (a) once we show that $J$ is the linear span of the $c_{i j}$ and the powers of $a$ and $b$. Let $L=A+B+C$ be this linear span where $A, B$, and $C$ denote respectively the linear span of the powers of $a, b$, and of the $c_{i j}$. Since $L$ contains the generators of $J$, it will be enough to prove that $L$ is a subalgebra of $J$ or that $L$ is invariant 
under all Jordan multiplications by elements of $J$. By [2, Proposition 3.2.4] the multiplications of $J$ are generated by the operators $V_{a}, V_{b}, U_{a}, U_{b}$, and $U_{a, b}$. By symmetry in $a$ and $b$ it therefore suffices to show that $L$ contains the following subspaces:
(i) $\quad V_{a} A+U_{a} A$,
(iv) $U_{a, b} A$,
(ii) $\quad V_{a} B$,
(v) $V_{a} C+U_{a} C$,
(iii) $U_{a} B$
(vi) $U_{a, b} C$.

The following well-known identities, valid in any unital Jordan algebra, will be needed [2, Chapter III]:

$$
\begin{gathered}
V\left(x^{i}\right) x^{j}=2 x^{i+j}, \quad U\left(x^{i}\right) x^{j}=x^{2 i+j}, \\
V\left(x^{i}, x^{j}\right)=V\left(x^{i+j}\right), \\
U\left(x^{i}, x^{j}\right)=V\left(x^{i}\right) V\left(x^{j}\right)-V\left(x^{i+j}\right), \\
U\left(x^{i+j}, x^{i}\right)=V\left(x^{j}\right) U\left(x^{i}\right)=U\left(x^{i}\right) V\left(x^{j}\right), \\
V(x, y) U_{x}=U\left(U_{x} y, x\right), \quad V(x, y) V_{x}=V\left(U_{x} y\right)+U_{x} V_{y} .
\end{gathered}
$$

In $J$ we now have

$$
U_{a} b^{i+1}=a \circ b^{i}-b^{i-1}
$$

for $i \geq 1$ and

$$
\begin{gathered}
\left\{a^{j} b^{i} a\right\}-\left\{a^{j+1} b^{i+1} a\right\}=c_{i-1, j}, \\
U_{a} c_{i j}=c_{i-1, j+1}, \\
V_{a} c_{i j}=c_{i-1, j}+c_{i, j+1},
\end{gathered}
$$

for $i, j \geq 0$. Indeed, (16) follows from $U_{a} b^{i+1}=U_{a} U_{b} b^{i-1}=V(a, b) b^{i-1}-$ $b^{i-1}($ by $(9))=V\left(b^{i-1}, b\right) a-b^{i-1}=b^{i} \circ a-b^{i-1}$ (by (12)). To prove (17) for $i \geq 1$ we use (13), (14), and (16):

$$
\begin{aligned}
\left\{a^{j} b^{i} a\right\}-\left\{a^{j+1} b^{i+1} a\right\} & =V\left(a^{j}\right) V_{a} b^{i}-V\left(a^{j+1}\right) b^{i}-V\left(a^{j}\right) U_{a} b^{i+1} \\
& =V\left(a^{j}\right)\left(a \circ b^{i}-U_{a} b^{i+1}\right)-a^{j+1} \circ b^{i} \\
& =V\left(a^{j}\right) b^{i-1}-a^{j+1} \circ b^{i}=c_{i-1, j},
\end{aligned}
$$

whereas for $i=0$ we obtain $a^{j} \circ a-\left\{a^{j+1} b a\right\}=a^{j} \circ a-V\left(a^{j}\right) U_{a} b=a^{j} \circ$ $\left(a-U_{a} b\right)=0=c_{-1, j}$ by (14) and (8). Formula (18) follows from (14) and (17):

$$
\begin{aligned}
U_{a} c_{i j} & =U_{a} V\left(a^{j}\right) b^{i}-U_{a} V\left(a^{j+1}\right) b^{i+1} \\
& =\left\{a^{j+1} b^{i} a\right\}-\left\{a^{j+2} b^{i+1} a\right\}=c_{i-1, j+1} .
\end{aligned}
$$

Finally, we prove (19) using (13) and (17):

$$
\begin{aligned}
V_{a} c_{i j} & =V_{a} V\left(a^{j}\right) b^{i}-V_{a} V\left(a^{j+1}\right) b^{i+1} \\
& =\left\{a^{j} b^{i} a\right\}+a^{j+1} \circ b^{i}-\left\{a^{j+1} b^{i+1} a\right\}-a^{j+2} \circ b^{i+1} \\
& =c_{i-1, j}+c_{i, j+1} .
\end{aligned}
$$

We can now show (10): (i) follows from (11); (ii) from $a \circ b^{n+1}=2 b^{n}-c_{n, 0}$ by definition of the $c_{i j}$; (iii) from $U_{a} b=a$, (ii), and (16); (iv) from $U_{a, b} a^{n}=$ $a^{n+1} \circ b=2 a^{n}-c_{0 n}$ by (12); and (v) from (18) and (19). Finally, for (vi) it 
suffices by the foregoing to prove $V_{a, b} C \subset L$ since $U_{a, b}=V_{a} V_{b}-V_{a, b}$. This follows from

$$
\left\{a b c_{i j}\right\}=\left(2-\delta_{0 j}\right) c_{i j}
$$

To show (20) for $j>0$, use (8), (15), and (18) to compute

$$
\left\{a b c_{i j}\right\}=V_{a, b} U_{a} c_{i+1, j-1}=U\left(U_{a} b, a\right) c_{i+1, j-1}=2 U_{a} c_{i+1, j-1}=2 c_{i j},
$$

whereas

$$
\begin{aligned}
\left\{a b c_{i 0}\right\} & =2\left\{a b b^{i}\right\}-V_{a, b} V_{a} b^{i+1} \\
& =2 a \circ b^{i+1}-V\left(U_{a} b\right) b^{i+1}-U_{a} V_{b} b^{i+1} \quad(\text { by }(12) \text { and }(15)) \\
& =a \circ b^{i+1}-2 U_{a} b^{i+2} \quad(\text { by }(8)) \\
& =a \circ b^{i+1}-2\left(a \circ b^{i+1}-b^{i}\right) \quad(\text { by }(16)) \\
& =c_{i 0} .
\end{aligned}
$$

This completes the proof.

Note that while there is associative asymmetry in $R$ as associative algebra (no automorphism switching $u, v$ ) there is complete Jordan symmetry between $u$ and $v$ in $R^{+}$.

Corollary 1. (a) $J$ is a free $k$-module with basis $1, a^{n}, b^{n} \quad(n \geq 1)$, and $c_{i j}=$ $a^{j} \circ b^{i}-a^{j+1} \circ b^{i+1}(i, j \geq 0)$, and $W_{a, b} J=0=W_{b, a} J$.

(b) For $i, j \geq 0$, the $c_{i j}$ satisfy the Jordan multiplication table for matrix units and the general Jordan shift formulas

$$
\begin{aligned}
U\left(a^{n}\right) c_{i j}=c_{i-n, j+n}, & U\left(b^{n}\right) c_{i j} & =c_{i+n, j-n}, \\
V\left(a^{n}\right) c_{i j}=c_{i-n, j}+c_{i, j+n}, & V\left(b^{n}\right) c_{i j} & =c_{i+n, j}+c_{i, j-n} .
\end{aligned}
$$

(c) The subspace $C$ spanned by the $c_{i j}$ is an ideal of $J$ and $J / C \cong$ $k\left[X, X^{-1}\right]^{+}$with $X=a+C, X^{-1}=b+C$.

Recall that $c_{p q}=0$ if either $p$ or $q$ is negative. For the proof, one uses $\varphi\left(c_{i j}\right)=e_{i j}, \varphi(a)=u, \varphi(b)=v$, and computes in $R$. (By the same method, it is easy to establish the complete multiplication table for the above basis of $J$.) Alternatively, (21) can be obtained from (18) and $U\left(x^{n}\right)=U(x)^{n}$, while for (22) one can argue by induction: $n=1$ is (19) and for $n>1$ one uses $V\left(a^{n}\right)=V_{a} V\left(a^{n-1}\right)-U_{a} V\left(a^{n-2}\right)$ by (13), (14).

Corollary 2. Let $A$ be a unital Jordan algebra containing noninvertible elements $x, y$ such that $U_{x} y=x, U_{y} x=y, W_{x, y}=W_{y, x}=0$. Then the elements $z_{i j}=x^{j} \circ y^{i}-x^{j+1} \circ y^{i+1}(i, j \geq 0)$ are nonzero Jordan matrix units. In particular, the $z_{i i}$ are nonzero orthogonal idempotents.

Proof. Let $\psi: J \rightarrow A$ be the unital homomorphism mapping $a \mapsto x, b \mapsto y$. Then $\psi\left(c_{i j}\right)=z_{i j}$. Assume $z_{m n}=0$ for some $m, n$. By applying $\psi$ to (21) and (22) one sees easily that $\psi\left(c_{i j}\right)=0$ for all $i, j \geq 0$. Hence $\psi$ factors via $J / C$. Since the canonical images of $a$ and $b$ in $J / C$ are inverses by Corollary $1(c)$, so are $x$ and $y$ in $A$, a contradiction.

Corollary 3. The assignment $a \mapsto s, b \mapsto t$ induces a Jordan homomorphism $\sigma: J \rightarrow S^{+}$which is an isomorphism between $J$ and $H(S, \pi)$, the set of $\pi$ hermitian elements in $S$. The unital special universal envelope of $J$ is isomorphic to $(S, \sigma)$. 
Proof. The formulas

$$
\sigma\left(a^{n}\right)=s^{n}, \quad \sigma\left(b^{n}\right)=t^{n}, \quad \sigma\left(c_{i j}\right)=g_{j i}+h_{i j}=h_{i j}+h_{i j}^{\pi}
$$

combined with Theorem 2(a) and Corollary $1(\mathrm{a})$ show that $\sigma$ is an isomorphism of $J$ with $H(S, \pi)$. (Note that, by Theorem $2(\mathrm{a}), H(S, \pi)$ is spanned by $1, s^{n}, t^{n}$, and $h_{i j}+h_{i j}^{\pi}$.) Let $\left(S^{\prime}, \sigma^{\prime}\right)$ be the unital special universal envelope of $J$. Then $\sigma=\eta \circ \sigma^{\prime}$ by the universal property of $S^{\prime}$ where $\eta: S^{\prime} \rightarrow S$ maps $s^{\prime}=\sigma^{\prime}(a)$ to $s$ and $t^{\prime}=\sigma^{\prime}(b)$ to $t$. On the other hand, an application of $\sigma^{\prime}$ to (8) and (9) shows that the elements $s^{\prime}, t^{\prime}$ of $S^{\prime}$ satisfy (1) and (2). Hence there is a homomorphism $\eta^{\prime}: S \rightarrow S^{\prime}$ sending $s$ to $s^{\prime}$ and $t$ to $t^{\prime}$, and clearly $\eta^{\prime}$ is the inverse of $\eta$.

\section{THE SHIFT TRIPLE SYSTEM}

We keep the notation of the previous section. The relations defining $J$ can be given a Jordan triple formulation as follows. Turn the $k$-module underlying $J$ into a Jordan triple system $J^{\tau}$ by setting $P_{x} y=U_{x} y^{\tau}$. Then $e=1_{J}$ and $f=a$ are tripotents (as is $\left.g=b=f^{\tau}\right)$ :

$$
e^{(3)}=e, \quad f^{(3)}=f,
$$

where $x^{(3)}=P_{x} x=U_{x} x^{\tau}$ denotes the third power in the triple sense. This is immediate from (6) and $a^{\tau}=b$. Also, $\tau=P_{e}$; whence, in particular,

$$
P_{e}^{2} f=f \text {. }
$$

Finally, the operators $W(x, y)$ in $J$ and $B(x, y)=\operatorname{Id}-L(x, y)+P_{x} P_{y}$ in $J^{\tau}$ (where we set $L(x, y) z=P(x+z) y-P_{x} y-P_{z} y=V\left(x, y^{\tau}\right) z$ ) are related by $B(x, y)=W\left(x, y^{\tau}\right)$. Since $J=\left(J^{\tau}\right)_{e}$ is the $e$-homotope of $J^{\tau}$ as a Jordan algebra, the powers $x^{n}$ in $J$ can be recovered in $J^{\tau}$ as the powers $x^{(n, e)}$ in the $e$-homotope of $J^{\tau}$, with the convention that $x^{(0, e)}=e$. Thus we obtain from (9) the relations

$$
B(f, f)\left(P_{e} f\right)^{(n, e)}=0 \text { for all } n \geq 0 .
$$

It is now natural to define the shift triple system as the Jordan triple system $T$ over $k$ presented by generators $e$ and $f$ and relations (23)-(25). We set $g=P_{e} f$.

We denote by $R^{*}$ the Jordan triple structure on $R$ given by $P_{x} y=x y^{*} x$.

Theorem 4. The assignments $e \mapsto 1_{J}, f \mapsto a$ and $e \mapsto 1_{R}, f \mapsto u$ respectively induce isomorphisms $T \cong J^{\tau}$ and $T \cong R^{*}$.

Proof. Clearly we have a homomorphism $\alpha: T \rightarrow J^{\tau}$ mapping $e$ to 1 and $f$ to $a$. To obtain a homomorphism in the opposite direction, we first observe that $T=T_{2}(e)$. Indeed, $T_{2}(e)$ is a subtriple containing the generators $e$ and $f$, by (24). Hence the $e$-homotope $T_{e}$ of $T$ is a Jordan algebra with unit element $e$, quadratic operators $U_{x}=P_{x} P_{e}$, and involutive automorphism $P_{e}$ interchanging $g$ and $f$. From (23)-(25) it follows easily that $f$ and $g$ satisfy (8) and (9) in $T_{e}$. This gives us a Jordan algebra homomorphism $\beta: J \rightarrow T_{e}$ with $\beta(1)=e, \beta(a)=f, \beta(b)=P_{e} f=g$. It follows that $\beta \circ \tau=P_{e} \circ \beta$; thus, $\beta: J^{\tau} \rightarrow T$ is a Jordan triple homomorphism which is the inverse of $\alpha$. Under the isomorphism $J \cong R^{+}$of Theorem 3 the involutions $\tau$ and ${ }^{*}$ correspond to each other. This proves the second statement. 
We leave it to the reader to formulate Corollary 1 of Theorem 3 in Jordan triple terms. Before proving an analogue of Corollary 2, we introduce the following terminology. A shift pair in a Jordan triple system $V$ is a pair $(c, d)$ of tripotents such that $d \in V_{2}(c)$ and

$$
V_{2}(c) \cap V_{0}(d)=0 \neq V_{2}(c) \cap V_{1}(d) .
$$

Note that (26) refers to all of $V_{2}(c)$, not just the part generated by $c$ and $d$. By $[3,1.8] c$ and $d$ have compatible Peirce decompositions. Hence (26) implies

$$
\begin{gathered}
V_{2}(c)=V_{2}(d) \oplus\left(V_{2}(c) \cap V_{1}(d)\right), \\
B(d, d) V_{2}(c)=0,
\end{gathered}
$$

since $B(d, d)$ is the projection onto $V_{0}(d)$. We note that, for compatible tripotents $c, d$ satisfying (28), the second condition of (26) is equivalent to either one of

$$
\left(P_{d}^{2}-\mathrm{Id}\right) V_{2}(c) \neq 0, \quad P_{d}^{2} c \neq c .
$$

Indeed, if $E_{i}$ are the projection operators onto the Peirce spaces of $d$, we have $V_{2}(c) \cap V_{1}(d)=E_{1}\left(V_{2}(c)\right)=\left(\mathrm{Id}-E_{0}-E_{2}\right) V_{2}(c)=\left(\mathrm{Id}-B(d, d)-P_{d}^{2}\right) V_{2}(c)=$ $\left(\right.$ Id $\left.-P_{d}^{2}\right) V_{2}(c)$, whence the first equivalence. Moreover, if $P_{d}^{2} c=c$ then $c \in$ $V_{2}(d)$ and so $V_{2}(c) \cap V_{1}(d) \subset V_{2}(d) \cap V_{1}(d)=0$, showing $V_{2}(c) \cap V_{1}(d) \neq 0$; hence, $P_{d}^{2} c \neq c$. Finally, $P_{d}^{2} c \neq c$ obviously implies $\left(P_{d}^{2}-\mathrm{Id}\right) V_{2}(c) \neq 0$.

Lemma 1. Let $(c, d)$ be a shift pair in the Jordan triple system $V$, and let $c=c_{2}+c_{1}$ be the Peirce decomposition of $c$ with respect to $d$. Then $c_{1}$ and $c_{2}$ are nonzero orthogonal tripotents. Furthermore, we have

$$
V_{2}\left(c_{1}\right) \subset V_{2}(c) \cap V_{1}(d),
$$

and $\left(d, c_{2}\right)$ is a shift pair.

Proof. By [3, 1.9] $c_{1}$ and $c_{2}$ are orthogonal tripotents. If $c_{1}=0$ then $c=c_{2} \in$ $V_{2}(d)$ implies $V_{2}(c) \subset V_{2}(d)$, contradicting (26) and (27). Likewise, $c_{2}=0$ yields $c=c_{1} \in V_{1}(d)$; hence, $P_{c} d \in V_{2}(c) \cap V_{0}(d)=0$ and thus $d=P_{c}^{2} d=0$, which again contradicts $(26)$.

From (27) and $c_{1} \in V_{2}(c) \cap V_{1}(d) \subset V_{2}(c)$ it follows by the Peirce relations that $V_{2}\left(c_{1}\right) \subset V_{2}(c)$ and

$$
\begin{aligned}
V_{2}\left(c_{1}\right)= & P\left(c_{1}\right) V_{2}(c) \subset P\left(c_{1}\right) V_{2}(d) \oplus P\left(c_{1}\right)\left(V_{1}(d) \cap V_{2}(c)\right) \\
& \subset\left(V_{0}(d) \cap V_{2}(c)\right) \oplus\left(V_{1}(d) \cap V_{2}(c)\right) .
\end{aligned}
$$

Since $V_{2}(c) \cap V_{0}(d)=0$ by $(26)$, this proves (29).

Finally, we must show

$$
V_{2}(d) \cap V_{0}\left(c_{2}\right)=0 \neq V_{2}(d) \cap V_{1}\left(c_{2}\right) .
$$

Since $c=c_{1}+c_{2}$ is an orthogonal decomposition, we have

$$
\begin{aligned}
& V_{0}\left(c_{2}\right) \cap V_{2}(c)=V_{2}\left(c_{1}\right) \cap V_{2}(c), \\
& V_{1}\left(c_{2}\right) \cap V_{2}(c)=V_{1}\left(c_{1}\right) \cap V_{2}(c) .
\end{aligned}
$$

Hence

$$
\begin{aligned}
V_{2}(d) \cap V_{0}\left(c_{2}\right)= & V_{2}(d) \cap V_{0}\left(c_{2}\right) \cap V_{2}(c) \\
= & V_{2}(d) \cap V_{2}\left(c_{1}\right) \cap V_{2}(c) \quad(\text { by }(30)) \\
& \subset V_{2}(d) \cap V_{1}(d) \quad(\text { by }(29)) \\
= & 0 .
\end{aligned}
$$


Assume $V_{2}(d) \cap V_{1}\left(c_{2}\right)=0$. Then $V_{2}(d)=V_{2}\left(c_{2}\right)$ since $V_{2}(d)$ is the sum of its intersections with the Peirce spaces of $c_{2}$. Thus $d$ and $c_{2}$ are associated and therefore have the same Peirce spaces; in particular, $V_{1}(d)=V_{1}\left(c_{2}\right)$. From (29) and (31) we get

$$
V_{2}\left(c_{1}\right) \subset V_{2}(c) \cap V_{1}\left(c_{2}\right)=V_{2}(c) \cap V_{1}\left(c_{1}\right) \subset V_{1}\left(c_{1}\right),
$$

which implies $c_{1}=0$, a contradiction.

Recall that two collinear tripotents $c, d$ are called rigidly collinear if $V_{2}(c) \subset$ $V_{1}(d)$ or, equivalently, $V_{2}(d) \subset V_{1}(c)$. Clearly, if $c, d$ are rigidly collinear then so are $c,-d$. Also recall that a rectangular $\left(N_{1} \times N_{2}\right)$-grid (cf. [5, II.2.3; 4]) in a Jordan triple system is a family $\mathscr{R}=\left(r_{i j}\right),(i, j) \in N_{1} \times N_{2}$, of nonzero tripotents such that the collinear pairs in $\mathscr{R}$ are those which have a first ("row") index or a second ("column") index in common, and $\left(r_{i j}, r_{i l}, r_{k l}, r_{k j}\right)$ is a quadrangle whenever $i \neq k$ and $j \neq l$. If any two collinear tripotents in $\mathscr{R}$ are rigidly collinear, we say $\mathscr{R}$ is rigid.

Lemma 2. (a) Let $\left(e_{1}, e_{2}, e_{3}, e_{4}\right)$ be a quadrangle of tripotents in a Jordan triple system $V$. If $e_{1}, e_{2}$ are rigidly collinear then so are $e_{i}, e_{i+1}$ for $i=2,3,4$ (indices $\bmod 4)$.

(b) Let $e_{1}, e_{2}, e_{3}$ be pairwise collinear tripotents. If $e_{1}, e_{2}$ are rigidly collinear so are $e_{1}, e_{3}$ and $e_{2}, e_{3}$.

(c) If a rectangular grid $\mathscr{R}=\left(r_{i j}\right)$ contains one rigidly collinear pair then $\mathscr{R}$ is rigid.

Proof. (a) It is enough to find automorphisms $\varphi_{i}, i=2,3,4$, of $V_{2}\left(e_{1}+e_{2}\right)$ $=V_{2}\left(e_{2}+e_{4}\right)$ mapping $\left\{e_{i}, e_{i+1}\right\}$ to $\left\{e_{1}, e_{2}\right\}$. Set $\varphi_{2}=P\left(e_{2}+e_{4}\right), \varphi_{3}=$ $P\left(e_{1}+e_{3}\right) P\left(e_{2}+e_{4}\right), \varphi_{4}=P\left(e_{1}+e_{3}\right)$.

(b) The automorphism $\theta=B\left(e_{1}+e_{3}, e_{1}+e_{3}\right)$ of $V[4,1.1]$ exchanges $e_{1}$ and $e_{3}$ and maps $e_{2}$ to $-e_{2}$, so $e_{2}$ and $e_{3}$ are rigidly collinear. By symmetry, the same holds for $e_{1}$ and $e_{3}$.

(c) Suppose that $e_{1}, e_{2}$ is a rigidly collinear pair in $\mathscr{R}$. By collinearity, we either have $\left(e_{1}, e_{2}\right)=\left(r_{i j}, r_{i l}\right)$ or $\left(e_{1}, e_{2}\right)=\left(r_{i j}, r_{k j}\right)$ for suitable indices $i, j, k, l$. It suffices to consider the first case. From the grid properties and (a) it follows that $A=\left\{r_{m j}: m \in N_{1}\right\}$ is a rigidly collinear family and from (b) that $B=\left\{r_{i n}: n \in N_{2}\right\}$ is also a rigidly collinear family. If $(c, d)$ is a collinear pair in $\mathscr{R}$ which is not contained in $A \cup B$ then there exist $e, f \in A \cup B$ such that $(c, d, e, f)$ is a quadrangle. Hence, again by $(\mathrm{a}),(c, d)$ is rigidly collinear.

We return to the notation introduced earlier and prove

Lemma 3. If $k$ is a field then $E$ is the unique minimal ideal of $R, R^{+}$, and $R^{*}$.

Proof. In $R^{*}$ we have $x^{*}=P_{1} x$; hence, every ideal of $R^{*}$ is *-invariant and thus an ideal of $R^{+}$. Clearly an ideal of $R$ is one of $R^{+}$. Therefore, it suffices to show that $E \subset K$ for every nonzero ideal $K$ of $R^{+}$. Since $E$ is simple as a Jordan algebra, it is even enough to prove $E \cap K \neq 0$. Suppose $x=\alpha_{0} \cdot 1+\sum_{l=1}^{p}\left(\alpha_{l} u^{l}+\alpha_{-l} v^{l}\right)+y \in K \backslash E$ where $y \in E$ so that $\alpha_{j} \neq 0$ for some $j$. In $R^{+}$we have the formulas $U\left(u^{n}\right) e_{i j}=e_{i-n, j+n}=0$ for $n>i$ and $u^{n} \circ e_{00}=e_{-n, 0}+e_{0, n}=e_{0, n}$ for $n>0\left(\mathrm{cf} .(21)\right.$ and (22)) and $U\left(u^{n}\right) v^{l}=u^{2 n-l}$ 
for $n \geq l$. Since $y$ is a finite linear combination of the $e_{i j}$, it follows that $U\left(u^{n}\right) y=0$ for sufficiently large $n$; thus, $x^{\prime}=U\left(u^{n}\right) x=\sum_{l=-p}^{p} \alpha_{l} u^{2 n+l} \in K$. Now $x^{\prime} \circ e_{00}=\sum_{l=-p}^{p} \alpha_{l} e_{0,2 n+l}$ is a nonzero element of $E \cap K$.

Theorem 5. Let $V$ be a Jordan triple system containing a shift pair $(c, d)$. Then the elements

$$
r_{i j}=\left\{d^{(j, c)}, c,\left(P_{c} d\right)^{(i, c)}\right\}-\left\{d^{(j+1, c)}, c,\left(P_{c} d\right)^{(i+1, c)}\right\} \quad(i, j \geq 0)
$$

form a rigid rectangular $(\mathbf{N} \times \mathbf{N})$-grid in $V$. If $k$ is a field, there exists a unique Jordan triple isomorphism $\psi$ from $T$ onto the subtriple generated by $c$ and $d$ such that $\psi(e)=c$ and $\psi(f)=d$.

Proof. From $d \in V_{2}(c)$ and (28) it follows that $c$ and $d$ satisfy (23)-(25). After identifying $T$ and $R^{*}$ by Theorem 4 , we have a Jordan triple homomorphism $\psi: R^{*} \rightarrow V$ mapping $1 \mapsto c$ and $u \mapsto d$. One checks easily that $\psi\left(e_{i j}\right)=r_{i j}$ using $\left(R^{*}\right)_{1}=R^{+}$, so $x^{(n, 1)}=x^{n}$ for every $x \in R$, $u^{j} \circ v^{i}=\left\{u^{i}, 1, v^{j}\right\}=\left\{u^{(j, 1)}, 1,\left(P_{1} u\right)^{(i, 1)}\right\}$, and $e_{i j}=u^{j} \circ v^{i}-u^{j+1} \circ v^{i+1}$. The $e_{i j}$ form a rectangular grid in $R^{*}$. Hence the $r_{i j}$ satisfy all grid relations, and therefore $r_{00} \neq 0$ implies $r_{i j} \neq 0$ (consider the quadrangle $\left(r_{00}, r_{0 j}, r_{i j}, r_{i 0}\right)$ ). To see that $r_{00} \neq 0$ we consider the Peirce decomposition of 1 in $R^{*}$, which is easily seen to be

$$
1=v u+e_{00} \in R_{2}^{*}(u) \oplus R_{1}^{*}(u),
$$

using $P_{u}^{2}(1)=P_{u}\left(u 1^{*} u\right)=P_{u}\left(u^{2}\right)=u v^{2} u=v u$ and $L(u, v) e_{00}=u v e_{00}+$ $e_{00} v u=e_{00}(1+v u)=e_{00}\left(2-e_{00}\right)=e_{00}$. By applying $\psi$ we get the Peirce decomposition $c=c_{2}+c_{1}$ of $c$ with respect to $d$ in $V$ where $c_{2}=\psi(v u)$ and $c_{1}=\psi\left(e_{00}\right)=r_{00}$ which is not zero by Lemma 1 .

Next we prove rigidity. By Lemma 2 it suffices to have $r_{00}$ and $r_{01}$ rigidly collinear. A computation shows that $v u$ is a tripotent in $R^{*}$ and that the Peirce decomposition of $u$ with respect to $v u$ is

$$
u=v u^{2}+e_{01} \in R_{2}^{*}(v u) \oplus R_{1}^{*}(v u)
$$

Indeed, $P_{v u}^{2} u=P_{v u}\left(v^{2} u\right)=v u^{2}$ and $L(v u, v u) e_{01}=v u v u e_{01}+e_{01} v u v u=$ $v u e_{01}+e_{01} v u=v u(1-v u) u+(1-v u) u v u=0+(1-v u) u=e_{01}$. An application of $\psi$ yields the Peirce decomposition $d=d_{2}+d_{1}$ with respect to $c_{2}=\psi(v u)$ in $V$, where $d_{1}=\psi\left(e_{01}\right)=r_{01}$. By Lemma $1,\left(d, c_{2}\right)$ is a shift pair. Hence (29), applied to $d, c_{2}, d_{1}$ in place of $c, d, c_{1}$ and (27), (31) imply

$$
\begin{aligned}
V_{2}\left(r_{01}\right) & =V_{2}\left(d_{1}\right) \subset V_{2}(d) \cap V_{1}\left(c_{2}\right) \subset V_{2}(c) \cap V_{1}\left(c_{2}\right) \\
& =V_{2}(c) \cap V_{1}\left(c_{1}\right) \subset V_{1}\left(c_{1}\right)=V_{1}\left(r_{00}\right) .
\end{aligned}
$$

It remains to prove that $\psi$ is injective if $k$ is a field. The kernel of $\psi$ is an ideal of $R^{*}$ not containing $E$ and hence is zero by Lemma 3. This completes the proof.

\section{CONCLUDING REMARKS}

(i) Suppose the base ring $k$ is a field. Then [1] the shift algebra $R$ is primitive and $E$ is the socle of $R$ and also the unique minimal ideal of $R$. Consequently, one shows as above that if $A$ is any associative unital $k$-algebra with two generators $x, y$ satisfying $x y=1 \neq y x$ then $A \cong R$. Similarly, one can prove that the symmetric shift algebra $S$ is semiprimitive but not primitive, 
with $G$ and $H$ as the only minimal ideals, and socle $G \oplus H$. The details are left to the reader.

(ii) From Lemma 1 and the proof of Theorem 5 one can deduce the following inductive construction of the tripotents $r_{i i}$ and $r_{i, i+1}$. Starting with a shift pair $(c, d)$, define two sequences $e_{n}, f_{n}$ of tripotents by $e_{0}=c, e_{1}=d$, and

$$
e_{n}=e_{n+2}+f_{n} \in V_{2}\left(e_{n+1}\right) \oplus V_{1}\left(e_{n+1}\right),
$$

the Peirce decomposition of $e_{n}$ with respect to $e_{n+1}$. Then $f_{0}, f_{1}, f_{2}, \ldots$ is the "infinite staircase" $r_{00}, r_{01}, r_{11}, r_{12}, \ldots$ in $\mathscr{R}$, from which $\mathscr{R}$ can be reconstructed by $[5$, II.2.6]. The details are omitted.

(iii) The exact sequence $0 \rightarrow E \rightarrow R \rightarrow k\left[X, X^{-1}\right] \rightarrow 0$ has the $C^{*}$-algebra analogue

$$
0 \rightarrow \mathscr{K} \rightarrow \mathscr{T} \rightarrow \mathscr{C}\left(S^{1}\right) \rightarrow 0
$$

where $\mathscr{T}$ denotes the Toeplitz algebra, $\mathscr{C}\left(S^{1}\right)$ the continuous functions on the circle, and $\mathscr{K}$ the compact operators on the Hardy space. More precisely, let $A$ be a $C^{*}$-algebra generated as a $C^{*}$-algebra by $u$ and $v$ satisfying $u v=1 \neq v u$ and $v=u^{*}$. Then one can show that $A$ and $\mathscr{T}$ are isomorphic as $C^{*}$ algebras. It might be interesting to explore this connection further, studying, on the one hand, more general shift algebras corresponding to the Toeplitz algebras of bounded symmetric domains [6] and, on the other hand, Toeplitz $J B^{*}$-triples.

\section{REFERENCES}

1. N. Jacobson, Some remarks on one-sided inverses, Proc. Amer. Math. Soc. 1 (1950), 352-355.

2. __ Structure theory of Jordan algebras, Univ. Arkansas Lecture Notes Math. Sci., vol. 5, Wiley, New York, 1981.

3. K. McCrimmon, Compatible Peirce decompositions of Jordan triple systems, Pacific J. Math. 103 (1982), 57-102.

4. K. McCrimmon and K. Meyberg, Coordinatization of Jordan triple systems, Comm. Algebra 9 (1981), 1495-1542.

5. E. Neher, Jordan triple systems by the grid approach, Lecture Notes in Math., vol. 1280, Springer, Berlin and New York, 1987.

6. H. Upmeier, Toeplitz operators and index theory in several complex variables, Proc. Sympos. Pure Math., vol. 51, Amer. Math. Soc., Providence, RI, 1990, pp. 585-598.

Institut für Mathematik, Universität INNSBRUCK, A-6020 INNSBRUCK, AUSTRIA

E-mail address: Ottmar.Loos@uibk.ac .at

Department of Mathematics, University of Ottawa, Ottawa, Ontario, Canada K1N 6N5

E-mail address: neher@acadvm1.uottawa.ca 